\title{
Dormant origins licensed by excess Mcm2-7 are required for human cells to survive replicative stress
}

\author{
Xin Quan Ge, ${ }^{1}$ Dean A. Jackson, ${ }^{2}$ and J. Julian Blow ${ }^{1,3}$ \\ ${ }^{1}$ Wellcome Trust Biocentre, University of Dundee, Dundee DD1 5EH, United Kingdom; ${ }^{2}$ Faculty of Life Sciences, \\ University of Manchester, Manchester Interdisciplinary Biocentre (MIB), Manchester, M1 7DN, United Kingdom
}

In late mitosis and early G1, Mcm2-7 complexes are loaded onto DNA to license replication origins for use in the upcoming $\mathrm{S}$ phase. However, the amount of $\mathrm{Mcm} 2-7$ loaded is in significant excess over the number of origins normally used. We show here that in human cells, excess chromatin-bound Mcm2-7 license dormant replication origins that do not fire during normal DNA replication, in part due to checkpoint activity. Dormant origins were activated within active replicon clusters if replication fork progression was inhibited, despite the activation of S-phase checkpoints. After lowering levels of chromatin-bound Mcm2-7 in human cells by RNA interference (RNAi), the use of dormant origins was suppressed in response to replicative stress. Although cells with lowered chromatin-bound Mcm2-7 replicated at normal rates, when challenged with replication inhibitors they had dramatically reduced rates of DNA synthesis and reduced viability. These results suggest that the use of dormant origins licensed by excess $M c m 2-7$ is a new and physiologically important mechanism that cells utilize to maintain DNA replication rates under conditions of replicative stress. We propose that checkpoint kinase activity can preferentially suppress initiation within inactive replicon clusters, thereby directing new initiation events toward active clusters that are experiencing replication problems.

[Keywords: Replication origins; dormant origins; Mcm2-7; genetic stability]

Supplemental material is available at http://www.genesdev.org.

Received September 21, 2007; revised version accepted October 23, 2007.

In order to preserve genomic stability, cells must completely replicate their genomes in each cell cycle. This requires that the two converging forks from each pair of adjacent origins progress until they meet each other and terminate. Individual forks can, however, irreversibly stall before termination. This can be caused by forks encountering DNA damage or tightly associated DNA-protein complexes, or by fork movement being slowed by replication inhibitors (Lambert and Carr 2005). Eukaryotic cells have evolved different mechanisms to maintain their genomic stability upon replication stress. One important response is the activation of checkpoint kinases (ATM, ATR, Chk1, and Chk2) that stabilize the forks, inhibit late origin firing, delay further progression through the cell cycle, and promote lesion repair (Bartek et al. 2004; Branzei and Foiani 2005; Lambert and Carr 2005).

Replication forks can only initiate at licensed origins. Origin licensing involves the coordinated action of ORC (origin recognition complex), Cdc6, and Cdt1 proteins

${ }^{3}$ Corresponding author.

E-MAIL j.j.blow@dundee.ac.uk; FAX 44-1383-388072.

Article is online at http://www.genesdev.org/cgi/doi/10.1101/gad.457807. that load Mcm2-7 complexes onto DNA (Blow and Dutta 2005). The Mcm2-7 complex is thought to form a clamp around DNA that provides helicase activity to unwind DNA ahead of the replication fork (Labib and Diffley 2001; Forsburg 2004). Origin licensing occurs only during late mitosis to early G1, and this restriction is essential to prevent DNA from replicating more than once in a single cell cycle (Blow and Dutta 2005). If two converging forks stall irreversibly during $\mathrm{S}$ phase and there is no licensed replication origin already present between them, the intervening DNA cannot be replicated since new origins cannot be licensed once $S$ phase has started.

Mcm2-7 complexes do not exclusively colocalize with sites of DNA synthesis (Madine et al. 1995; Krude et al. 1996; Dimitrova et al. 1999). Furthermore, Mcm2-7 complexes are loaded onto DNA in an $\sim 20$-fold excess over replication origins and ORC (Burkhart et al. 1995; Lei et al. 1996; Rowles et al. 1996; Donovan et al. 1997; Mahbubani et al. 1997; Edwards et al. 2002) and are distributed at significant distances away from where ORC is bound (Ritzi et al. 1998; Edwards et al. 2002; Harvey and Newport 2003). Cells continue to synthesize DNA normally when the level of Mcm2-7 is reduced (Cortez 
et al. 2004; Tsao et al. 2004) and in Xenopus egg extracts normal replication rates are maintained when $M c m 2-7$ levels are reduced to approximately two per origin (Mahbubani et al. 1997; Edwards et al. 2002; Oehlmann et al. 2004; Woodward et al. 2006). Various suggestions have been made for the function of the excess Mcm2-7 complexes, including roles in DNA pumping (Laskey and Madine 2003), checkpoint activation (Cortez et al. 2004; Tsao et al. 2004), transcriptional regulation (Yankulov et al. 1999; DaFonseca et al. 2001; Fitch et al. 2003), and chromatin remodeling (Burke et al. 2001; Dziak et al. 2003).

A recent study has shown that most of the Mcm2-7 loaded onto DNA in Xenopus egg extracts could support the initiation of replication if normal S-phase levels of checkpoint activity were artificially inhibited by caffeine (Woodward et al. 2006). If artificially relieved from this checkpoint suppression by caffeine, the use of these additional "dormant" origins allowed efficient replication to occur in the presence of DNA polymerase inhibitors and DNA damage. However, the need to abolish checkpoint activity in these experiments makes their physiological relevance unclear.

Here, we describe the role of $\mathrm{Mcm} 2-7$ in licensing dormant origins and their usage in a physiological context when checkpoint responses remain intact. We show that human tissue culture cells contain dormant origins that are suppressed by normal levels of S-phase checkpoint activity, but are utilized when replication forks are inhibited despite S-phase checkpoint activation. These dormant origins were suppressed when chromatinbound Mcm2-7 levels were lowered approximately fourfold by small interfering RNA (siRNA), and this left cells hypersensitive to replication inhibitors. We conclude that dormant origins provide an important new mechanism used by human cells to maintain genome stability.

\section{Results}

\section{Hydroxyurea (HU) and aphidicolin promote firing of additional origins}

In animal cells, clusters of approximately three to eight adjacent replication origins fire together, with different clusters being activated at different times. In order to estimate the density of active origins in human U2OS cells, we used DNA fiber analysis, which involves labeling nascent DNA in vivo and then visualizing DNA molecules after they have been spread on microscope slides. In one protocol, cells were pulsed with BrdU, and clusters containing at least four consecutive BrdU tracks were chosen for analysis (see example in Fig. 1A). The track lengths within individual clusters were similar (correlation ratio 0.494), as expected if the origins fired together. The mean fork spacing within each cluster was then derived from the measurements of the distances between the center points of all the tracks in each cluster. Figure 1B shows the distribution of mean intracluster center-to-center distances for normal U2OS cells.
Overall fork spacing was $\sim 25 \mathrm{~kb}$, implying a mean replicon size of $\sim 50 \mathrm{~kb}$ within clusters, consistent with previous work (Berezney et al. 2000).

Cells were then treated with HU, an inhibitor of ribonucleotide reductase that lowers dNTP pools and thereby inhibits DNA synthesis. After $4 \mathrm{~h}$ of $200 \mu \mathrm{M}$ $\mathrm{HU}$, the mean replication fork spacing had reduced to $\sim 17 \mathrm{~kb}$ (Fig. 1B, gray bars). In addition, the correlation ratio of track lengths within each cluster dropped to 0.393 , suggesting that origin firing within the cluster had become less synchronous, as would be expected if dormant origins had been induced to fire after forks from the primary origins had been inhibited. A titration of HU revealed that the fork spacing decreased markedly from $50 \mu \mathrm{M}$ to $200 \mu \mathrm{M}$ HU (Fig. 1C). Similar results were obtained using aphidicolin, an inhibitor of replicative DNA polymerases (Supplementary Fig. S1).

There is some ambiguity in the interpretation of the fork density data shown in Figure 1, B and C, because it is not possible to determine the direction of fork movement. To confirm our conclusions, we therefore used a double-labeling strategy as shown in Figure 1D. Cells were first pulsed with BrdU and then with biotin-dUTP; after spreading, antibodies specific to the two labels can then be used to show the direction of fork movement, which allows the positions of the replication origins to be approximated. This technique gave a mean origin-toorigin spacing of $49 \mathrm{~kb}$ in U2OS cells (Fig. 1E), very close to the value of $\sim 50 \mathrm{~kb}$ implied by the single-labeling protocol. When cells were treated with $200 \mu \mathrm{M} H U$, the distance between adjacent replication origins fell to 25 $\mathrm{kb}$, also in agreement with the results obtained from the single-labeling experiments (Fig. 1B). We repeated the double-labeling analysis in nontransformed MRC5 cells, which again showed a marked reduction in origin spacing when cells were treated with $200 \mu \mathrm{M}$ HU (Supplementary Fig. S2). Our results are also consistent with earlier data suggesting that when DNA synthesis is inhibited in animal cells, additional replication origins fire that otherwise remain dormant (Ockey and Saffhill 1976; Taylor 1977; Francis et al. 1985; Painter 1985; Griffiths and Ling 1987; Anglana et al. 2003; Gilbert 2007).

These results are surprising because replication inhibitors activate checkpoint kinases such as Chk1 (Fig. 1C, inset) that suppress further initiation from late firing origins (Dimitrova and Gilbert 2000; Feijoo et al. 2001; Merrick et al. 2004). To investigate the role that checkpoint kinases play in the response to HU, we knocked down the Chk1 protein level using siRNA (Supplementary Fig. S4A; Rocha et al. 2005). Figure 1F shows that when Chk1 levels were knocked down, replication fork spacing was reduced from a mean of $\sim 25 \mathrm{~kb}$ to $\sim 14 \mathrm{~kb}$. This is consistent with results suggesting that inhibition of checkpoint systems leads to an increase in origin firing (Lehmann 1972; Syljuasen et al. 2005; Woodward et al. 2006; Maya-Mendoza et al. 2007). HU was able to reduce fork spacing even further in the Chk1 knockdown cells (Fig. 1F, dashed outline). Similar results were obtained when checkpoint kinases were inhibited using caffeine, 
an ATM/ATR inhibitor (Supplementary Fig. S3B). This suggests that $\mathrm{HU}$ increases origin usage in response to replicative stress by a mechanism that does not depend on checkpoint kinases.
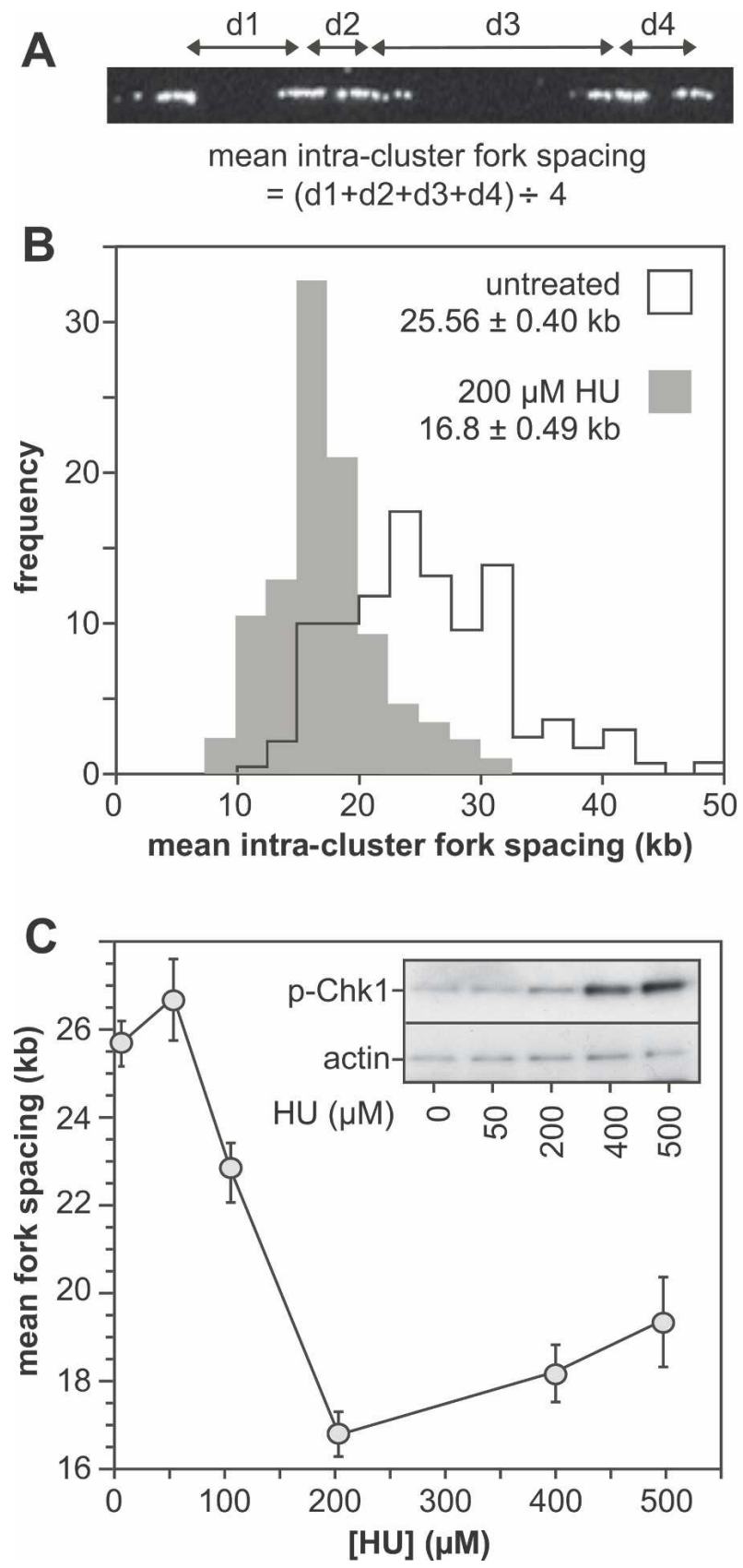

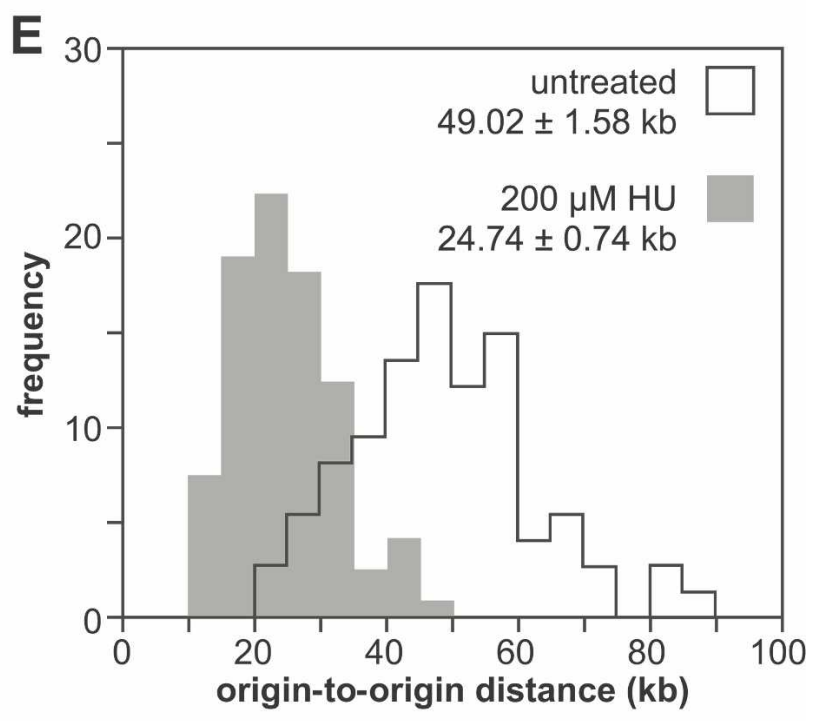

Dormant origins depend on Mcm2-7 levels

If dormant replication origins are licensed by excess Mcm2-7 on DNA, then reducing chromatin-bound Mcm2-7 should counteract the reduction in fork spacing
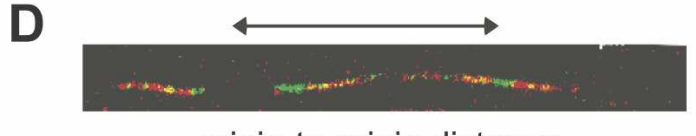

origin-to-origin distance

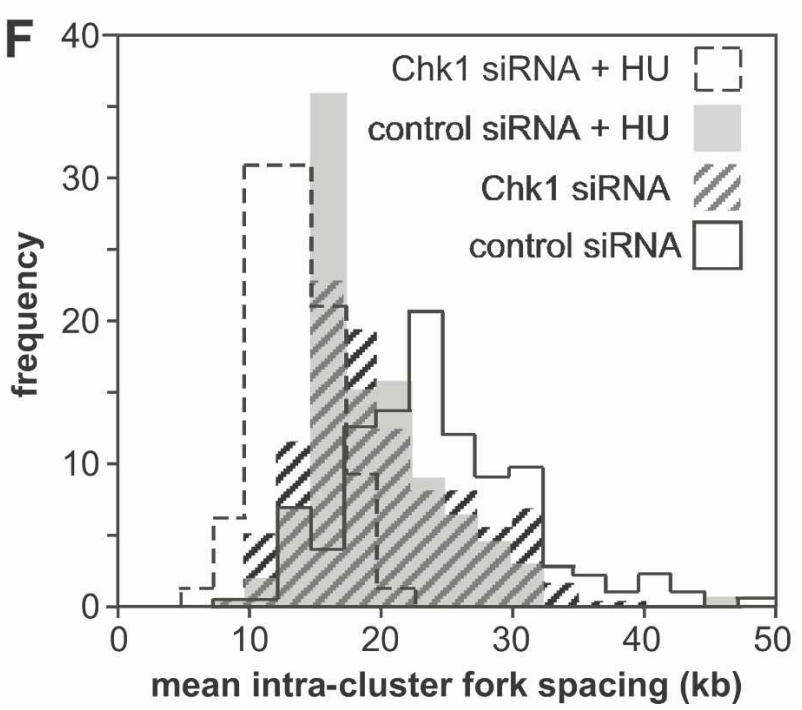

Figure 1. HU activates the firing of additional origins. $(A-C) \mathrm{U} 2 \mathrm{OS}$ cells were incubated $\pm \mathrm{HU}$ for $4 \mathrm{~h}$ and pulsed with BrdU. DNA was isolated and spread on glass slides, and BrdU-labeled tracks were detected with fluorescent antibodies. Clusters containing four or more tracks were selected. $(A)$ The distance between the center points of adjacent tracks in a cluster was measured and averaged to give the mean intracluster fork spacing. $(B)$ The mean intracluster fork spacings were determined for at least $100 \mathrm{clusters} \pm 200 \mu \mathrm{M} \mathrm{HU}$. (C) The mean intracluster fork spacings were averaged to give mean fork spacing in each sample $\pm S E M$ for the indicated concentrations of $H U$. (Inset) Cells treated with 0-500 $\mu \mathrm{M}$ HU for $4 \mathrm{~h}$ were immunoblotted for phospho-Chk1 and actin. $(D, E)$ U2OS cells were incubated $\pm \mathrm{HU}$ for $4 \mathrm{~h}$ and pulsed with BrdU followed by biotin-dUTP. (D) DNA was isolated and spread on glass slides, and labeled tracks were detected with fluorescent antibodies. $(E)$ The distribution of origin-to-origin distances were determined $\pm 200 \mu M H U$; the overall mean and SEM are also indicated. $(F)$ Mean intracluster fork spacing in cells treated with Chk1 siRNA or control siRNA for 96 h, followed by $\pm 200 \mu \mathrm{M}$ HU for $4 \mathrm{~h}$. Overall means \pm SEM (in kilobases) are control, $24.663 \pm 0.511$, control+ HU, $16.08 \pm 0.33 ; \mathrm{Chk} 1,20.42 \pm 0.38$; Chk1 + HU, $13.71 \pm 0.29$. 
Ge et al.

A
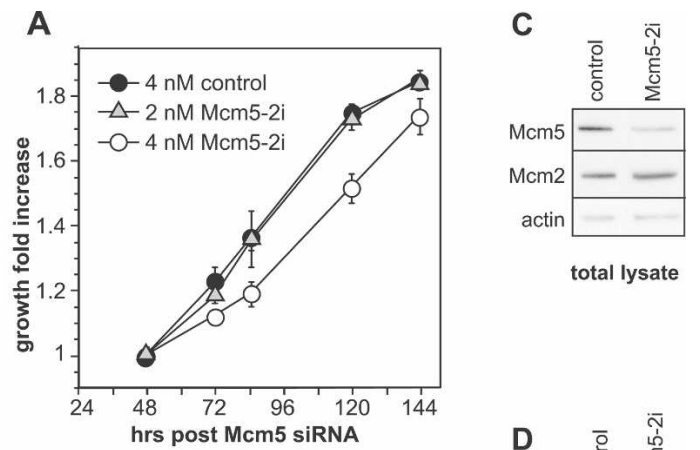

total lysate
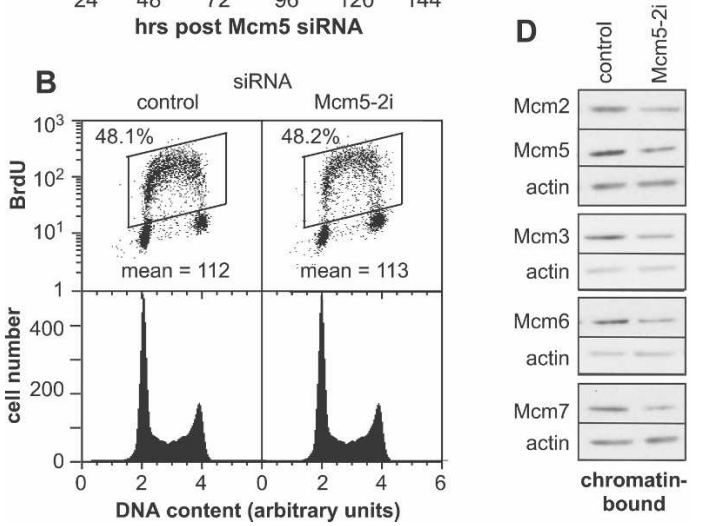

Figure 2. Mcm5 knockdown cells proliferate and replicate DNA normally. (A) U2OS cells were transfected with 2 or $4 \mathrm{nM}$ Mcm5-2i siRNA or $4 \mathrm{nM}$ control siRNA and their proliferation rate was analyzed 48-144 h after transfection. $(B-D)$ Analysis was carried out $96 \mathrm{~h}$ after $2 \mathrm{nM}$ Mcm5-2i siRNA. $(B)$ Cells were pulsed with BrdU for $30 \mathrm{~min}$, and their rate of DNA synthesis as well as DNA content were analyzed by FACS. The percentage S-phase cells and their mean BrdU incorporation are indicated. (C) Immunoblot showing the levels of total Mcm5 and $\mathrm{Mcm} 2$. (D) Immunoblot showing the levels of chromatin-bound Mcm2, Mcm3, Mcm5, Mcm6, and Mcm7.

caused by HU. We therefore used siRNAs targeting different regions of MCM5 mRNA to knock down MCM5 expression in U2OS cells. siRNAs were titrated to find the maximum concentration that maintained normal proliferation rates. Figure 2A shows the effect of one particular MCM5 siRNA (oligo MCM5-2i) on total cell proliferation. This siRNA ( $2 \mathrm{nM}$ ) was chosen for subsequent experiments as it maintained normal cell proliferation. Cells treated with $2 \mathrm{nM}$ MCM5-2i had a normal cell cycle distribution and maintained normal rates of BrdU incorporation (Fig. 2B). Although total Mcm5 levels were reduced to $\sim 25 \%$ (Fig. 2C), levels of chromatin-bound Mcm2, Mcm3, Mcm5, Mcm6, and Mcm7 were all reduced by $\sim 50 \%$ (Fig. $2 \mathrm{D}$ ), suggesting that lowering the levels of Mcm5 caused a corresponding decrease in the ability of the entire Mcm2-7 complex to be loaded onto chromatin (Prokhorova and Blow 2000). A similar knockdown was also obtained with a different oligo, MCM5-1i (Supplementary Fig. S4).

DNA fiber analysis showed that the MCM5-2i siRNA treatment caused no significant increase in average origin spacing (Fig. 3A) or fork spacing (Fig. 3C). The correlation ratio of track lengths within each cluster was similar in cells treated with either control or Mcm5-2i siRNA (0.488 and 0.478 , respectively), suggesting that Mcm5 knockdown had not affected the synchrony of origin firing within individual clusters. However, when the MCM5-2i-treated cells were exposed to $200 \mu \mathrm{M} H U$, they were unable to increase origin or fork density to the same degree as cells treated with control siRNA (Fig. $3 \mathrm{~B}, \mathrm{D})$. This demonstrates that reducing the quantity of $\mathrm{Mcm} 5$ on chromatin reduces the number of dormant origins that can be activated in response to replicative stress. In the presence of $200 \mu \mathrm{M} \mathrm{HU}$, the correlation ratio of track lengths within each cluster in cells treated with Mcm5-2i siRNA fell to only 0.430, compared with 0.354 in control cells. This is consistent with the Mcm5 knockdown cells being unable to fully activate dormant origins after replication forks have been slowed by HU, so that there is less asynchrony in origin firing than in cells treated with control siRNA.

Since the firing of dormant origins is suppressed by checkpoint kinases, we investigated the effect of partial Mcm5 knockdown on replication fork spacing in cells
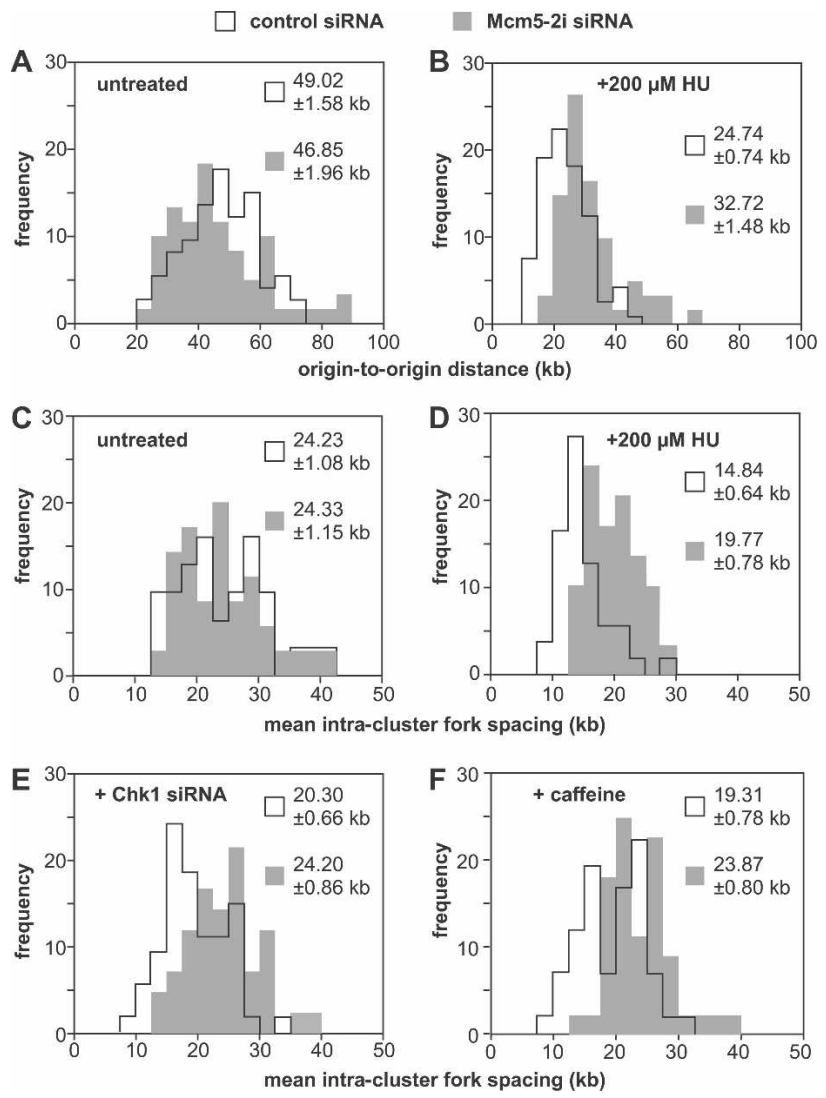

Figure 3. Excess $\mathrm{Mcm} 2-7$ are required for dormant origin firing. U2OS cells were transfected with $2 \mathrm{nM} \mathrm{Mcm5-2i} \mathrm{siRNA} \mathrm{or}$ control siRNA optionally plus Chk1 siRNA. Ninety-six hours later they were optionally treated for $4 \mathrm{~h}$ with $200 \mu \mathrm{M} H \mathrm{HU}$ or for $2 \mathrm{~h}$ with $5 \mathrm{mM}$ caffeine. $(A, B)$ Cells were pulsed with BrdU and then biotin-dUTP, and interorigin distances were measured. (C$F$ ) Cells were pulsed with BrdU, and intracluster fork spacing was measured. The mean and standard error of the mean for each sample are indicated. 
where checkpoint kinases were inhibited. Figure 3, E and F, shows that treatment of cells with MCM5-2i siRNA suppressed the ability of cells to reduce replication fork spacing when checkpoint kinases were inhibited by Chk1 siRNA or caffeine. Similar results were obtained with another Mcm5 siRNA (Mcm5-1i), though in this case treatment with the Mcm5 siRNA alone caused a slight increase in fork spacing (Supplementary Fig. S5). These results are consistent with the idea that dormant origins licensed by excess Mcm2-7 are normally suppressed by checkpoint kinases but can be activated when cells experience replicative stress, and that partial knockdown of Mcm5 by siRNA strongly reduces the number of available dormant origins.

\section{Mcm5 knockdown cells are hypersensitive to $H U$}

We next investigated the rate of cellular DNA synthesis in Mcm5 knockdown cells by measuring BrdU incorporation (Fig. 4A). In the absence of $\mathrm{HU}$, replication rates in Mcm5 knockdown cells were similar to control cells, but after treatment with $200 \mu \mathrm{M}$ HU for either 4 or $40 \mathrm{~h}$, the Mcm5 knockdown cells replicated their DNA much more slowly than control cells. This effect was observed over a range of HU concentrations (Fig. 4B; Supplementary Fig. S6) and was also seen using the MCM5-1i siRNA (Supplementary Fig. S6). This suggests that the full complement of dormant origins is required for cells to maintain replication rates in the presence of $\mathrm{HU}$.

After $40 \mathrm{~h}$ in $200 \mu \mathrm{M}$ HU, Mcm5 knockdown cells showed a markedly different cell cycle profile to control cells, with more cells in S and G2, and fewer cells in G1 (Fig. 4A). The Mcm5 knockdown cells showed increased levels of chromatin-bound PCNA, ubiquitinated PCNA, RPA, and DNA polymerase $\delta$ (Fig. 4C), as expected of cells experiencing replicative stress. The Mcm5 knockdown cells exposed to $200 \mu \mathrm{M}$ HU also had increased levels of p53 and p53 phosphorylated on Ser15, suggesting activation of cellular stress response pathways, though levels of phospho-Chk1, phospho-Rad17, or phospho-H2AX were similar (Fig. 4D). This is consistent with the idea that Mcm5 knockdown cells experience difficulty in completing DNA synthesis in the presence of HU and so accumulate in S phase with damaged DNA.

We next investigated how the Mcm5 knockdown cells behave over longer times after HU treatment (Fig. 5A). Mcm5 knockdown and control cells were treated for 48 $\mathrm{h}$ with different concentrations of $\mathrm{HU}$, and cell numbers were then determined. Figure 5B shows that HU reduced the proliferation of Mcm5 knockdown cells more strongly than control cells. Long-term survival was assessed by treating Mcm5 knockdown and control cells with HU for $48 \mathrm{~h}$ and then allowing surviving cells to form colonies in the absence of HU. Figure 5, C and D, shows that even in the absence of HU, the Mcm5 knock-
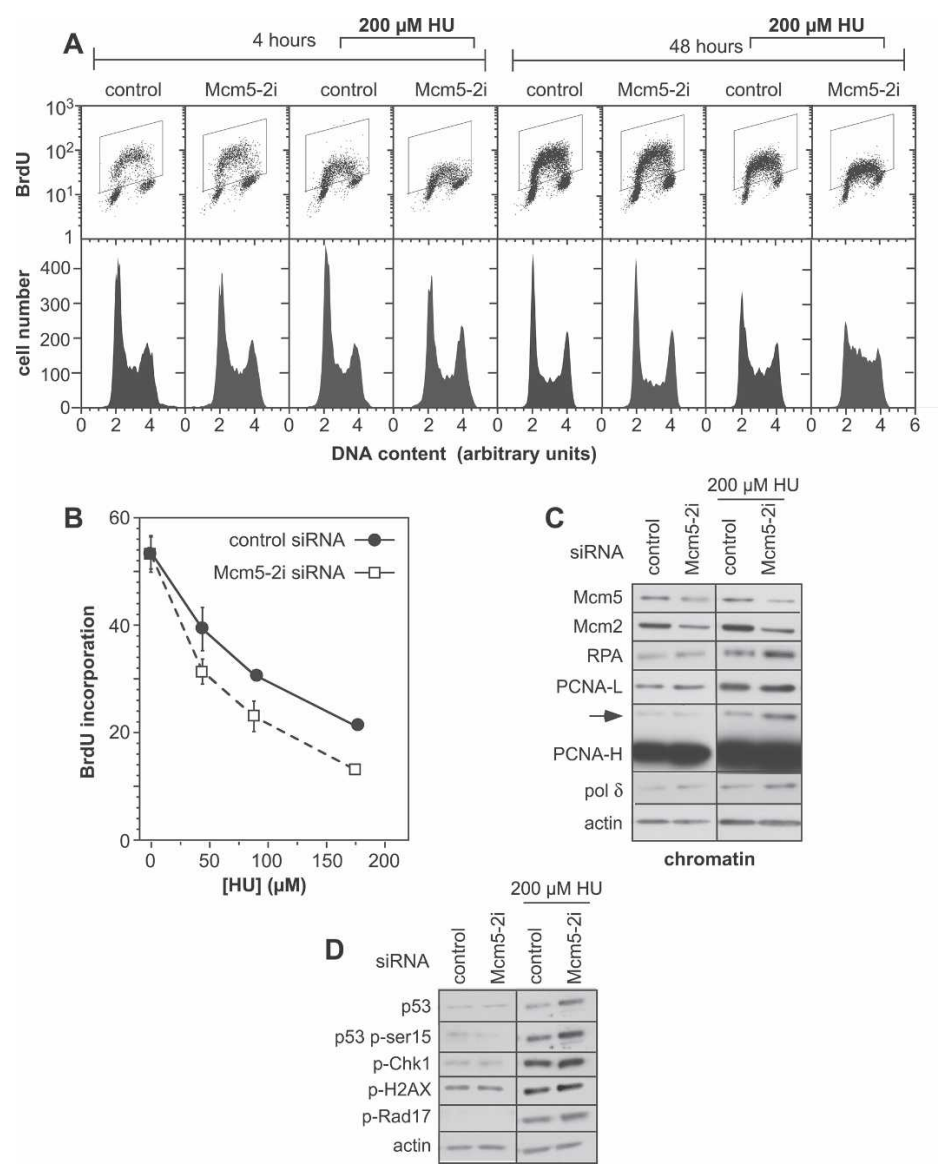

Figure 4. Mcm5 knockdown reduces DNA synthesis in response to HU. Cells were transfected with 2 nM Mcm5-2i siRNA. $(A, B)$ Cells were incubated in $\mathrm{HU}$ for $4 \mathrm{~h}$ (starting $92 \mathrm{~h}$ after siRNA) or $40 \mathrm{~h}$ (starting $56 \mathrm{~h}$ after siRNA), followed by a 30 -min BrdU pulse. (A) HU was used at $200 \mu \mathrm{M}$. DNA and BrdU content were analyzed by FACS. $(B)$ Total BrdU incorporation after 4 -h treatment with HU. $(C, D)$ Fifty hours post-siRNA transfection, cells were incubated ${ }_{ \pm} \mathrm{HU}$ for $48 \mathrm{~h}$ then immunoblotted for the indicated checkpoint proteins in isolated chromatin $(C)$ or whole-cell lysates $(D)$. PCNA-L is a light exposure of a PCNA blot to show relative PCNA levels; PCNA-H is a heavy exposure of the same blot, showing an additional band (arrow) migrating at the position of ubiquitinated PCNA. 
Ge et al.

Figure 5. Mcm5 knockdown cells are hypersensitive to HU. Cells were treated with $2 \mathrm{nM}$ Mcm5-2i siRNA or control siRNA. Forty-eight hours or $72 \mathrm{~h}$ later they were treated with HU for a further $48 \mathrm{~h}$. Cell number was then assessed; alternatively, cells were subject to a colony-forming assay. (A) Time scale of assays. (B) Cell number was measured $96 \mathrm{~h}$ after siRNA and was expressed relative to the cell number plated $40 \mathrm{~h}$ after siRNA. $(C, D)$ Surviving colonies were examined $2 \mathrm{wk}$ after siRNA and HU treatment. $(C)$ An example of colonies $\pm 0.5 \mathrm{mM} \mathrm{HU}$ treatment. $(D)$ Quantification of colony numbers.
A
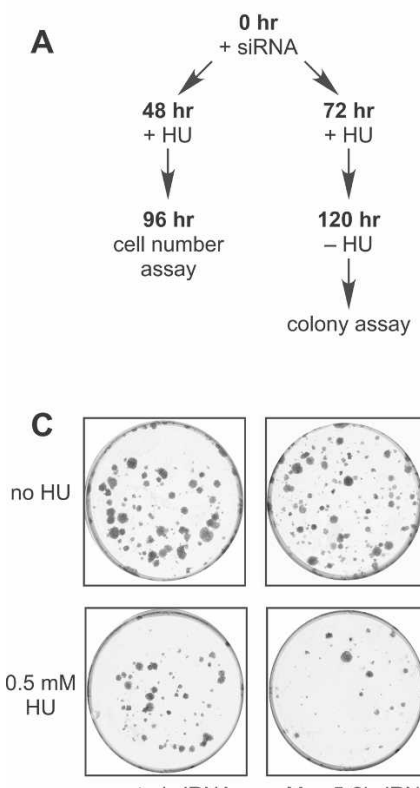

control siRNA
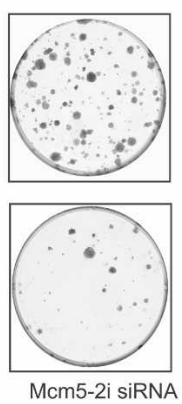
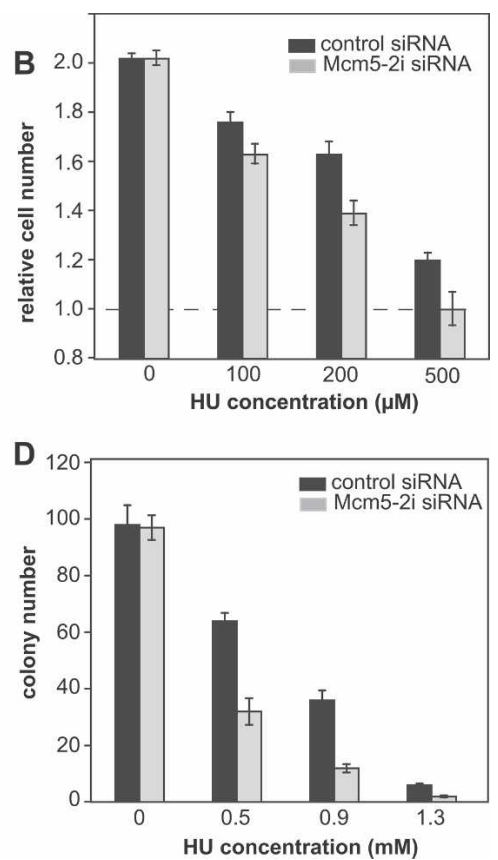

down cells formed slightly fewer colonies than control cells, suggesting that endogenous levels of replicative stress may have reduced the long-term survival of Mcm5 knockdown cells. When cells were transiently exposed to $\mathrm{HU}$, the survival of the Mcm5 knockdown cells dropped even further compared with the control cells (Fig. 5C,D). These results suggest that dormant origins are required to enhance survival in response to exogenous and endogenous replicative stresses.

\section{Mcm5 knockdown cells are hypersensitive to other replication stresses}

Finally, we investigated whether Mcm5 knockdown cells were hypersensitive to replication stresses other than HU. Aphidicolin inhibits replicative DNA polymerases and activates dormant origins (Supplementary Fig. S1). Camptothecin traps topoisomerase I in a covalent complex with DNA, thereby blocking replication forks that collide with it. Mcm5 knockdown cells showed a profound accumulation in late S and G2 when treated with low concentrations of aphidicolin or camptothecin (Fig. 6A). Compared with control cells, Mcm5 knockdown cells also displayed higher levels of p53 activation (Fig. 6B) and a decreased ability to form colonies after treatment with the inhibitors (Fig. 6C,D). These effects were also seen with a different anti-Mcm5 siRNA (Supplementary Fig. S7). These results are all consistent with the idea that the use of dormant origins is required for cells to survive a range of replicative stresses.

\section{Discussion}

In this paper, we show that treatment of human U2OS cells with inhibitors of DNA replication induced the fir- ing of additional "dormant" replication origins within origin clusters. During a normal S phase, firing of these dormant origins was suppressed by low levels of endogenous S-phase checkpoint kinases, but in response to replication inhibition dormant origins were activated to maintain DNA replication rates despite the increased checkpoint activity. The initiation of these dormant origins was also sensitive to the partial reduction of chromatin-bound Mcm2-7 complex by Mcm5 RNA interference (RNAi), suggesting that they are licensed by the excess Mcm2-7 loaded onto chromatin in G1. Although cells with a reduced chromatin level of Mcm2-7 showed normal rates of progression through $S$ phase, DNA synthesis, proliferation, and cell survival were all highly sensitive to replication inhibitors. These experiments suggest that the usage of dormant origins licensed by Mcm2-7 represents an important new pathway employed by mammalian cells to maintain genetic stability.

This work is consistent with considerable prior literature suggesting that replication origin spacing can be reduced by replication inhibitors (Ockey and Saffhill 1976; Taylor 1977; Francis et al. 1985; Painter 1985; Griffiths and Ling 1987; Anglana et al. 2003; Gilbert 2007) or by checkpoint inhibition (Lehmann 1972; Syljuasen et al. 2005; Woodward et al. 2006; Maya-Mendoza et al. 2007). In particular, our work extends the results of Woodward et al. (2006), who used caffeine-induced inhibition of checkpoint kinases in Xenopus egg extract to show that most of the Mcm2-7 loaded onto chromatin prior to $S$ phase could be used as replication origins. Woodward et al. (2006) also showed that when checkpoint responses were abolished, the use of these dormant origins could help maintain high rates of DNA synthesis in the presence of replicative stress. However, the need to abolish checkpoint activity to observe activation of dormant ori- 

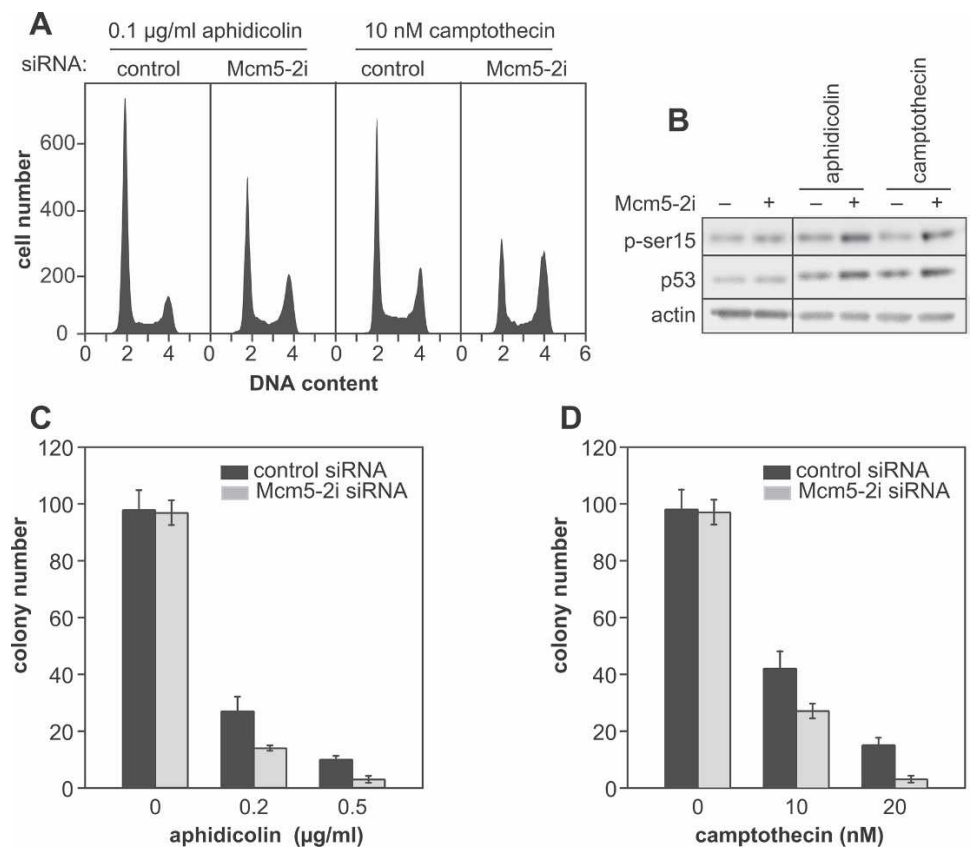

Figure 6. Mcm5 knockdown causes hypersensitivity to aphidicolin and camptothecin. $(A, B)$ Fifty hours posttransfection with $2 \mathrm{nM}$ Mcm5-2i siRNA or control siRNA, cells were incubated with $0.1 \mu \mathrm{g} / \mathrm{mL}$ aphidicolin or $10 \mathrm{nM}$ camptothecin for $48 \mathrm{~h}$. DNA content was measured by FACS $(A)$ and whole-cell lysates were immunoblotted for p53, p53 phospho-Ser15, and actin $(B)$. $(C, D)$ The clonogenic assay described in Figure 5A was applied to cells treated with aphidicolin $(C)$ or camptothecin $(D)$.

gins in these experiments meant that their relevance to replication inhibition in normal cells was unclear. The work described here in human cells shows that replication can initiate at dormant origins in response to replication stress even in the presence of checkpoint pathway activation. This strongly suggests that dormant origin usage is a physiologically important response of cells to replication inhibition.

\section{Dormant origins depend on the full complement of Mcm2-7}

Mcm2-7 are loaded onto chromatin to license origins of replication in late mitosis and G1. It is important that origin licensing is restricted to this cell cycle period, as the presence of $\mathrm{Mcm} 2-7$ is used to mark DNA that has not been replicated in the current cell cycle (Blow and Dutta 2005). Therefore, cells cannot license new origins during $S$ phase in response to replication problems, as the newly licensed origins would be created on both replicated DNA and unreplicated DNA, leading to rereplication of DNA. It therefore seems sensible for cells to license more origins in G1 than would normally be needed as a precautionary measure should problems arise during the subsequent $\mathrm{S}$ phase.

In animal cells, many replication origins (probably the majority) (Mesner et al. 2006) take the form of broad initiation zones, each representing an array of different sites where replication can initiate (DePamphilis 1999; Gilbert 2001; Machida et al. 2005). In any given S phase, a cell will usually use only one or two of these sites (Dijkwel et al. 2002), which is sometimes referred to as the "Jesuit model" of replication origins (DePamphilis 1999|. Mcm2-7 are likely to define the sites where replication can initiate and are distributed at different sites throughout initiation zones (Alexandrow et al. 2002). Consistent with this idea, we show that a fourfold knockdown of Mcm5 significantly reduced the activation of dormant origins within origin clusters in response to replication inhibition or checkpoint inhibition. Because dormant origins will be more effective the more widely distributed they are, our hypothesis that additional (dormant) origins are used when replication is inhibited provides an explanation of why metazoan replication origins are distributed in initiation zones.

Although strongly supporting a role for $\mathrm{Mcm} 2-7$ in licensing dormant origins of replication, our results do not rule out additional roles for $\mathrm{Mcm} 2-7$ in other processes such as checkpoint activation (Cortez et al. 2004; Tsao et al. 2004) or transcriptional activation (Yankulov et al. 1999; DaFonseca et al. 2001; Fitch et al. 2003). However, we saw no evidence for a defect in checkpoint signaling in Mcm5 knockdown cells. Importantly, if Chk1 activation were significantly compromised in the Mcm5 knockdown cells, we would have seen an increase in dormant origin firing rather than the decrease that was actually observed.

\section{Regulation of dormant origin usage}

The activation of dormant origins by replicative stress does not require a special mechanism, so long as origin firing is stochastic. Once an origin cluster becomes activated, dormant origins within that cluster normally have only a brief time to fire before they are passively replicated (and hence inactivated) by a fork from a neighboring origin. When fork progression is slowed by an inhibitor or DNA damage, dormant origins are more likely to fire because there is an increased period of time before they are passively replicated (Fig. 7). Consistent 
with this "passive" mechanism, we show that dormant origin firing does not depend on checkpoints, since HU still induced the firing of additional dormant origins in the absence of checkpoint kinase activity. Support for a passive mechanism also comes from the similarity in fork slowing (two- to threefold) and the increased origin density (approximately twofold) induced by $200 \mu \mathrm{M}$ HU. Furthermore, the synchrony of origin firing within clusters (as inferred from track length) was reduced in cells treated with HU (as expected if dormant origins were induced to fire only after forks from the primary origins had been slowed), and this fall was less pronounced in Mcm5 knockdown cells.

Despite increasing the density of replication forks within active origin clusters, $\mathrm{HU}$ reduced the total rate of cellular DNA synthesis. Since the average replicon in a cluster would be fully replicated in only 15-40 min and we examined replication kinetics $4 \mathrm{~h}$ after $\mathrm{HU}$ addition, this implies that the total number of actively initiating clusters was reduced. This is expected if checkpoint kinases activated by replicative stress inhibit the initiation of later-firing replication origins (Santocanale and Diffley 1998; Shirahige et al. 1998; Feijoo et al. 2001; Luciani et al. 2004; Merrick et al. 2004; Syljuasen et al. 2005). Consistent with other reports (Woodward et al. 2006; Maya-Mendoza et al. 2007), we show that inhibition of

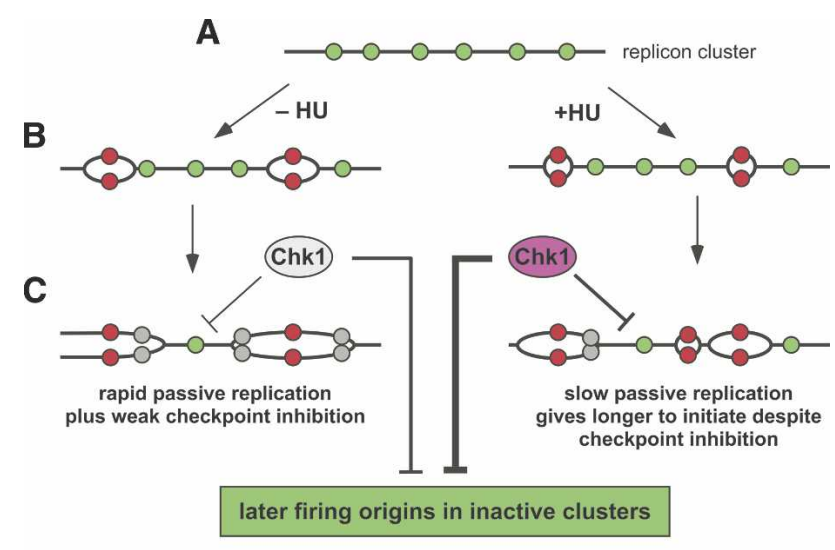

$$
\begin{aligned}
& \text { - Enfired, licensed origin } \\
& - \text { - Fired origin } \\
& - \text { Passively replicated origin }
\end{aligned}
$$

Figure 7. Models of how replicative stress could activate dormant origins. Unfired replication origins licensed by $\mathrm{Mcm} 2-7$ (green circles) are shown distributed on DNA (black line) within a replicon cluster. Origins can become inactivated (unlicensed) as a consequence either of initiating (red circles) or of being passively replicated (gray circles). (A) A cluster before it has become activated for initiation. $(B)$ The cluster soon after activation in the absence (left) or presence (right) of HU. (C) By slowing down fork progression, $\mathrm{HU}$ (or another replication inhibitor) gives dormant origins more time to fire, despite the increased repression of initiation by checkpoint kinases such as Chk1. However, Chk1 represses initiation more strongly in later-firing (inactive) clusters than in clusters that are already active. checkpoint kinases also increases the initiation of dormant origins. Taken together, these results suggest that although checkpoint kinases suppress initiation in both inactive, later-firing clusters and in active clusters, the inactive clusters are more strongly repressed (Fig. 7C). The trade-off between passive activation and checkpoint suppression makes dormant origins in active clusters fire preferentially, as demonstrated by the observation that dormant origin firing was maximal when replication rates were slowed less than threefold and Chk1 was only slightly activated. This suggests that at low levels of replicative stress, the intra-S-phase checkpoint does not simply suppress all new initiation, but instead diverts new initiation events away from replicon clusters that have not yet fired and stimulates initiation within currently active clusters.

Although the activation of dormant origins can be explained by this passive process, we cannot rule out the possibility of there being an active process that links replicative stress or fork stalling to the activation of dormant origins. All the forks associated with a replicon cluster are thought to colocalize in a single replication factory, and this might provide a physical structure that could promote initiation at nearby dormant origins if other forks in the factory stalled.

\section{Dormant origins are needed for cells to survive replicative stress}

Although they supported normal replication rates in the absence of exogenous replication inhibitors, cells with a partial knockdown of Mcm5 were unable to maintain high rates of DNA synthesis in the presence of DNA synthesis inhibitors. Mcm5 knockdown cells also showed an enhanced activation of a range of DNA damage and checkpoint markers. This suggests that cells are dependent on dormant origins to efficiently replicate DNA when forks are slowed or stalled.

When Mcm5 knockdown cells were transiently exposed to HU, their long-term survival was significantly compromised, suggesting that dormant origins are required to prevent the build-up of lethal genetic defects. Consistent with this, a recent report has shown that a destabilizing mutation of MCM4 makes mice highly susceptible to chromosome breaks induced by aphidicolin and prone to mammary adenocarcinomas (Shima et al. 2007). Interestingly, although their short-term proliferation was normal, our partial Mcm5 knockdown cells showed a reduced long-term viability even in the absence of exogenous replication inhibitors. This suggests that cells might also need dormant origins to deal with replication problems that occur during normal cell cycles.

Cells have evolved a complex series of mechanisms to maintain their genetic integrity during progression through the cell cycle. These include fidelity mechanisms acting at the replication fork, DNA repair pathways, and checkpoint systems that deal with DNA damage or replication fork stalling. We can now add dormant replication origins to this list as another important 
mechanism by which cells deal with replicative stress. It is tempting to speculate that the differing abilities of cells to deal with replicative stress (such as differences between normal and cancer cells) might be due to differences in the number of dormant origins that they can use.

\section{Materials and methods}

\section{Cells lines and siRNA}

U2OS cells were grown in DMEM medium containing $10 \%$ fetal bovine serum, penicillin, and streptomycin. siRNA oligonucleotides (Ambion) were made to the following sequences (sense-strand): Mcm5-1i, 5'-GGAGGUAGCUGAUGAGGUG TT-3'; Mcm5-2i, 5'-GGAUCUGGCCAGCUUUGAUTT-3'; Chk1, 5'-AAGCAGUCGCAGUGAAGAUUG-3' (Rocha et al. 2005).

Control-unrelated oligo was purchased from Dharmacon. Transfections were performed using Oligofectamine (Invitrogen), according to the manufacture's protocol. For Chk1 knockdown, U2OS were transfected with $40 \mathrm{nM}$ siRNA twice within $72 \mathrm{~h}$. siRNA transfection efficiency was $\sim 85 \%$.

\section{Antibodies and reagents}

Antibodies used in this report were anti-Chk1 (sc-8408, Santa Cruz Biotechnoloty), phospho-Chk1 Ser345 (2341, Cell Signaling), phospho-Chk2 Thr68 (2661, Cell Signaling), phosphoRad17 Ser645 (BL236, Bethyl), phospho- $\gamma$-H2AX Ser139 (JBW301, Upstate Biotechnology), phospho-p53 Ser15 (9284, Cell Signaling), p53 (DO-1, Santa Cruz Biotechnology), actin (ACTN05, Labvision), RPA70 (BL915, Bethyl), PCNA (PC10, Cell Signaling), Mcm2 (BM28, BD Biosciences), and Mcm5 (CRCT5.1, Labvision). The DNA polymerase $\delta$ polyclonal antibody was raised against a fragment of the Xenopus protein and cross-reacted with a human protein of the anticipated size (G. Stewart and J.J. Blow, in prep.). Aphidicolin (Sigma), camptothecin (Sigma), and nocodazole (Sigma) were prepared as stock in DMSO, while caffeine and HU (Sigma) were made freshly in $\mathrm{H}_{2} \mathrm{O}$ before use. Caffeine was used at $5 \mathrm{mM}$ to inhibit checkpoint signaling.

\section{Immunoblotting}

To prepare chromatin samples, $10^{6}$ cells washed with PBS were suspended in $2 \mathrm{~mL}$ of cytoskeleton (CSK) buffer (Dimitrova et al. 1999) and incubated on ice for $10 \mathrm{~min}$. After centrifugation at $5000 \mathrm{~g}$ for $5 \mathrm{~min}$, the pellet was collected, resuspended in CSK buffer, and incubated for $10 \mathrm{~min}$ on ice. After a second 5-min spin at $5000 g$, the resultant pellet containing chromatin-bound sample was washed with CSK buffer three times and suspended in $100 \mu \mathrm{L}$ of Nupage LDS sample buffer (Invitrogen, supplemented with $5 \% 2$-mercaptoethanol). To analyze total cellular proteins, $10^{5}$ cells were washed with PBS and suspended in 100 $\mu \mathrm{L}$ of LDS sample buffer. SDS gel electrophoresis and Western blotting were performed according to standard procedures.

\section{Immunofluorescence}

Immunostaining for total Mcm5 protein was as described (Tsao et al. 2004), except fixation was performed with $2 \%$ paraformaldehyde for 10 min and blocking was with PBS containing 5\% BSA and $0.5 \%$ Triton X-100. Mcm5 was detected with mAb (2 $\mu \mathrm{g} / \mathrm{mL}$ ) followed by Alexa 555-conjugated anti-mouse IgG (Molecular Probes).

\section{FACS}

BrdU-7AAD double-labeling was performed as described (Ekholm-Reed et al. 2004). Briefly, asynchronous cells were pulsed with $10 \mu \mathrm{M}$ BrdU (Sigma) for $30 \mathrm{~min}$, acid-treated, and detected using FITC-conjugated anti-BrdU antibody (556028, BD Biosciences). DNA was stained with $20 \mu \mathrm{g} / \mathrm{mL} 7 \mathrm{AAD}$. For phospho$\mathrm{H} 3$ detection, cells were fixed in $1 \%$ paraformaldehyde for 20 min at $37^{\circ} \mathrm{C}$ followed by $70 \%$ ice-cold ethanol. Phospho-H3 was detected with FITC-conjugated antibody $(3 \mathrm{H} 10$, Upstate Biotechnologyl and DNA was stained with $20 \mu \mathrm{g} / \mathrm{mL}$ 7AAD. For DNA content analysis, cells were fixed in $70 \%$ ethanol and stained with $50 \mu \mathrm{g} / \mathrm{mL}$ propidium iodide as described (Tsao et al. 2004). All samples were analyzed using a FACSCalibur flow cytometer (BD Biosciences) and CellQuest software. To quantify BrdU incorporation, the geographical mean of incorporated BrdU was then calculated (since the data are collected on a logarithmic scale), from which the overall rate of DNA synthesis was derived.

\section{Cell proliferation and clonogenic assays}

Forty-eight hours after siRNA transfection, cells were reseeded in a 96-well plate and their proliferation was assessed afterward every $24 \mathrm{~h}$ for $4 \mathrm{~d}$ with the 3-(4,5-dimethylthiazol-2-yl)-2,5-diphenyltetrazolium bromide-like colorimetric alamarBlue assay, which is based on the detection of metabolic activity (Biosource International). Absorbance was measured at 570 and $595 \mathrm{~nm}$. For clonogenic assay, cells were plated $48 \mathrm{~h}$ post-siRNA transfection on six-well plate in triplicate with 500 cells per well and incubated for 2 wk to allow colonies to form. Colonies were detected by crystal violet staining.

\section{DNA fiber spreads and analysis}

For single-label fork density measurements, cells were pulsed with $40 \mu \mathrm{M}$ BrdU for different times depending on the concentration of inhibitor used so that labeled track lengths were more homogenous in size than would occur if the BrdU pulse length were kept constant: $10 \mathrm{~min}$ for $0-100 \mu \mathrm{M}$ HU, 20 min for 200 $\mu \mathrm{M}$ HU, $30 \mathrm{~min}$ for $400-500 \mu \mathrm{M} \mathrm{HU}, 20 \mathrm{~min}$ for $0.1-0.2 \mu \mathrm{g} / \mathrm{mL}$ aphidicolin, and $30 \mathrm{~min}$ for $0.4 \mu \mathrm{g} / \mathrm{mL}$ aphidicolin. For duallabeling origin spacing measurements, cells were pulse-labeled with $10 \mu \mathrm{M}$ BrdU, washed, and then transfected with $33 \mu \mathrm{M}$ biotin-11-dUTP. Cells were then harvested and DNA fiber spreads were prepared as described (Jackson and Pombo 1998). BrdU-labeled tracks were detected with BrdU anti-sheep antibody (1:1000; M20105S, Biodesign) using either Cy3-conjugated or AlexaFluor-488-conjugated donkey anti-sheep secondary antibody. Biotin-11-dUTP was detected using a mouse monoclonal antibody (1:1000 dilution; Clone BN-34, Sigma) and appropriate Cy3-conjugated second antibody. Quality control for spreading DNA was performed using YOYO $0.1 \mu \mathrm{M}$; Molecular Probes) labeling. Fibers were examined using a Leica microscope (65× lens) and Zeiss LSM 510 confocal microscope (100× lens).

The mean and standard deviation of track lengths were first determined by measuring the length of labeled tracks that were well separated from other tracks (thereby minimizing the risk that they represented fusions between adjacent replicons). Track clusters were then selected for the determination of intracluster fork density and origin spacing by the following criteria: (1) Clusters consisted of single DNA fibers and not fiber bundles based on YOYO staining; (2) clusters were located in a 
relatively isolated area; (3) clusters contained at least four consecutive tracks; (4) each track in the cluster was no longer than the mean track length +1 standard deviation, to minimize the risk of including clusters where termination and fusion of neighboring replicons had occurred. For each sample, at least 100 measurements were performed. The correlation ratio $(\eta)$ for intracluster track length was also calculated as the variance of the mean track lengths for individual clusters divided by the variance of the entire population of track lengths.

\section{Acknowledgments}

Thanks to Apolinar Maya-Mendoza for his help with the fiber spreads, and to Tomo Tanaka and John Rouse for comments on the manuscript. This work was supported by CR-UK UK studentship C303/A4416 (X.G.), CR-UK Grant C303/A3135 (J.J.B.), and BBSRC grant B/06091 (D.A.J.).

\section{References}

Alexandrow, M.G., Ritzi, M., Pemov, A., and Hamlin, J.L. 2002. A potential role for mini-chromosome maintenance (MCM) proteins in initiation at the dihydrofolate reductase replication origin. J. Biol. Chem. 277: 2702-2708.

Anglana, M., Apiou, F., Bensimon, A., and Debatisse, M. 2003. Dynamics of DNA replication in mammalian somatic cells: Nucleotide pool modulates origin choice and interorigin spacing. Cell 114: 385-394.

Bartek, J., Lukas, C., and Lukas, J. 2004. Checking on DNA damage in S phase. Nat. Rev. Mol. Cell Biol. 5: 792-804.

Berezney, R., Dubey, D.D., and Huberman, J.A. 2000. Heterogeneity of eukaryotic replicons, replicon clusters, and replication foci. Chromosoma 108: 471-484.

Blow, J.J. and Dutta, A. 2005. Preventing re-replication of chromosomal DNA. Nat. Rev. Mol. Cell Biol. 6: 476-486.

Branzei, D. and Foiani, M. 2005. The DNA damage response during DNA replication. Curr. Opin. Cell Biol. 17: 568-575.

Burke, T.W., Cook, J.G., Asano, M., and Nevins, J.R. 2001. Replication factors MCM2 and ORC1 interact with the histone acetyltransferase HBO1. J. Biol. Chem. 276: 15397-15408.

Burkhart, R., Schulte, D., Hu, D., Musahl, C., Gohring, F., and Knippers, R. 1995. Interactions of human nuclear proteins P1Mcm3 and P1Cdc46. Eur. J. Biochem. 228: 431-438.

Cortez, D., Glick, G., and Elledge, S.J. 2004. Minichromosome maintenance proteins are direct targets of the ATM and ATR checkpoint kinases. Proc. Natl. Acad. Sci. 101: 1007810083.

DaFonseca, C.J., Shu, F., and Zhang, J.J. 2001. Identification of two residues in MCM5 critical for the assembly of MCM complexes and Stat1-mediated transcription activation in response to IFN- $\gamma$. Proc. Natl. Acad. Sci. 98: 3034-3039.

DePamphilis, M.L. 1999. Replication origins in metazoan chromosomes: Fact or fiction? Bioessays 21: 5-16.

Dijkwel, P.A., Wang, S., and Hamlin, J.L. 2002. Initiation sites are distributed at frequent intervals in the Chinese hamster dihydrofolate reductase origin of replication but are used with very different efficiencies. Mol. Cell. Biol. 22: 30533065.

Dimitrova, D.S. and Gilbert, D.M. 2000. Temporally coordinated assembly and disassembly of replication factories in the absence of DNA synthesis. Nat. Cell Biol. 2: 686-694.

Dimitrova, D.S., Todorov, I.T., Melendy, T., and Gilbert, D.M. 1999. Mcm2, but not RPA, is a component of the mammalian early G1-phase prereplication complex. J. Cell Biol. 146: 709-722.
Donovan, S., Harwood, J., Drury, L.S., and Diffley, J.F. 1997. Cdc6p-dependent loading of Mcm proteins onto pre-replicative chromatin in budding yeast. Proc. Natl. Acad. Sci. 94: 5611-5616.

Dziak, R., Leishman, D., Radovic, M., Tye, B.K., and Yankulov, K. 2003. Evidence for a role of MCM (mini-chromosome maintenance 5 in transcriptional repression of sub-telomeric and Ty-proximal genes in Saccharomyces cerevisiae. J. Biol. Chem. 278: 27372-27381.

Edwards, M.C., Tutter, A.V., Cvetic, C., Gilbert, C.H., Prokhorova, T.A., and Walter, J.C. 2002. MCM2-7 complexes bind chromatin in a distributed pattern surrounding the origin recognition complex in Xenopus egg extracts. J. Biol. Chem. 277: 33049-33057.

Ekholm-Reed, S., Mendez, J., Tedesco, D., Zetterberg, A., Stillman, B., and Reed, S.I. 2004. Deregulation of cyclin E in human cells interferes with prereplication complex assembly. J. Cell Biol. 165: 789-800.

Feijoo, C., Hall-Jackson, C., Wu, R., Jenkins, D., Leitch, J., Gilbert, D.M., and Smythe, C. 2001. Activation of mammalian Chk1 during DNA replication arrest: A role for Chk1 in the intra-S phase checkpoint monitoring replication origin firing. J. Cell Biol. 154: 913-923.

Fitch, M.J., Donato, J.J., and Tye, B.K. 2003. Mcm7, a subunit of the presumptive MCM helicase, modulates its own expression in conjunction with Mcm1. J. Biol. Chem. 278: 2540825416.

Forsburg, S.L. 2004. Eukaryotic MCM proteins: Beyond replication initiation. Microbiol. Mol. Biol. Rev. 68: 109-131.

Francis, D., Davies, N.D., Bryant, J.A., Hughes, S.G., Sibson, D.R., and Fitchett, P.N. 1985. Effects of psoralen on replicon size and mean rate of DNA synthesis in partially synchronized cells of Pisum sativum L. Exp. Cell Res. 158: 500-508.

Gilbert, D.M. 2001. Making sense of eukaryotic DNA replication origins. Science 294: 96-100.

Gilbert, D.M. 2007. Replication origin plasticity, Taylor-made: Inhibition vs. recruitment of origins under conditions of replication stress. Chromosoma 116: 341-347.

Griffiths, T.D. and Ling, S.Y. 1987. Activation of alternative sites of replicon initiation in Chinese hamster cells exposed to ultraviolet light. Mutat. Res. 184: 39-46.

Harvey, K.J. and Newport, J. 2003. CpG methylation of DNA restricts prereplication complex assembly in Xenopus egg extracts. Mol. Cell. Biol. 23: 6769-6779.

Jackson, D.A. and Pombo, A. 1998. Replicon clusters are stable units of chromosome structure: Evidence that nuclear organization contributes to the efficient activation and propagation of S phase in human cells. J. Cell Biol. 140: 1285-1295.

Krude, T., Musahl, C., Laskey, R.A., and Knippers, R. 1996. Human replication proteins hCdc21, hCdc46 and $\mathrm{P} 1 \mathrm{Mcm} 3$ bind chromatin uniformly before S-phase and are displaced locally during DNA replication. J. Cell Sci. 109: 309-318.

Labib, K. and Diffley, J.F. 2001. Is the MCM2-7 complex the eukaryotic DNA replication fork helicase? Curr. Opin. Genet. Dev. 11: 64-70.

Lambert, S. and Carr, A.M. 2005. Checkpoint responses to replication fork barriers. Biochimie 87: 591-602.

Laskey, R.A. and Madine, M.A. 2003. A rotary pumping model for helicase function of MCM proteins at a distance from replication forks. EMBO Rep. 4: 26-30.

Lehmann, A.R. 1972. Effect of caffeine on DNA synthesis in mammalian cells. Biophys. J. 12: 1316-1325.

Lei, M., Kawasaki, Y., and Tye, B.K. 1996. Physical interactions among $\mathrm{Mcm}$ proteins and effects of $\mathrm{Mcm}$ dosage on DNA replication in Saccharomyces cerevisiae. Mol. Cell. Biol. 16: 5081-5090. 
Luciani, M.G., Oehlmann, M., and Blow, J.J. 2004. Characterization of a novel ATR-dependent, Chk1-independent, intraS-phase checkpoint that suppresses initiation of replication in Xenopus. J. Cell Sci. 117: 6019-6030.

Machida, Y.J., Hamlin, J.L., and Dutta, A. 2005. Right place, right time, and only once: Replication initiation in metazoans. Cell 123: 13-24.

Madine, M.A., Khoo, C.Y., Mills, A.D., Musahl, C., and Laskey, R.A. 1995. The nuclear envelope prevents reinitiation of replication by regulating the binding of MCM3 to chromatin in Xenopus egg extracts. Curr. Biol. 5: 1270-1279.

Mahbubani, H.M., Chong, J.P., Chevalier, S., Thömmes, P., and Blow, J.J. 1997. Cell cycle regulation of the replication licensing system: Involvement of a Cdk-dependent inhibitor. J. Cell Biol. 136: 125-135.

Maya-Mendoza, A., Petermann, E., Gillespie, D.A., Caldecott, K.W., and Jackson, D.A. 2007. Chk1 regulates the density of active replication origins during the vertebrate $S$ phase. EMBO T. 26: 2719-2731.

Merrick, C.J., Jackson, D., and Diffley, J.F. 2004. Visualization of altered replication dynamics after DNA damage in human cells. J. Biol. Chem. 279: 20067-20075.

Mesner, L.D., Crawford, E.L., and Hamlin, J.L. 2006. Isolating apparently pure libraries of replication origins from complex genomes. Mol. Cell 21: 719-726.

Ockey, C.H. and Saffhill, R. 1976. The comparative effects of short-term DNA Inhibition on replicon synthesis in mammalian cells. Exp. Cell Res. 103: 361-373.

Oehlmann, M., Score, A.J., and Blow, J.J. 2004. The role of Cde6 in ensuring complete genome licensing and $\mathrm{S}$ phase checkpoint activation. J. Cell Biol. 165: 181-190.

Painter, R.B. 1985. Inhibition and recovery of DNA synthesis in human cells after exposure to ultraviolet light. Mutat. Res. 145: 63-69.

Prokhorova, T.A. and Blow, J.J. 2000. Sequential MCM/P1 subcomplex assembly is required to form a heterohexamer with replication licensing activity. J. Biol. Chem. 275: 2491-2498.

Ritzi, M., Baack, M., Musahl, C., Romanowski, P., Laskey, R.A., and Knippers, R. 1998. Human minichromosome maintenance proteins and human origin recognition complex 2 protein on chromatin. J. Biol. Chem. 273: 24543-24549.

Rocha, S., Garrett, M.D., Campbell, K.J., Schumm, K., and Perkins, N.D. 2005. Regulation of NF- $\mathrm{kB}$ and p53 through activation of ATR and Chk1 by the ARF tumour suppressor. EMBO I. 24: 1157-1169.

Rowles, A., Chong, J.P., Brown, L., Howell, M., Evan, G.I., and Blow, J.J. 1996. Interaction between the origin recognition complex and the replication licensing system in Xenopus. Cell 87: 287-296.

Santocanale, C. and Diffley, J.F. 1998. A Mec1- and Rad53-dependent checkpoint controls late-firing origins of DNA replication. Nature 395: 615-618.

Shima, N., Alcaraz, A., Liachko, I., Buske, T.R., Andrews, C.A., Munroe, R.J., Hartford, S.A., Tye, B.K., and Schimenti, J.C. 2007. A viable allele of $\mathrm{Mcm} 4$ causes chromosome instability and mammary adenocarcinomas in mice. Nat. Genet. 39: 93-98.

Shirahige, K., Hori, Y., Shiraishi, K., Yamashita, M., Takahashi, K., Obuse, C., Tsurimoto, T., and Yoshikawa, H. 1998. Regulation of DNA-replication origins during cell-cycle progression. Nature 395: 618-621.

Syljuasen, R.G., Sorensen, C.S., Hansen, L.T., Fugger, K., Lundin, C., Johansson, F., Helleday, T., Sehested, M., Lukas, J., and Bartek, J. 2005. Inhibition of human Chk1 causes increased initiation of DNA replication, phosphorylation of ATR targets, and DNA breakage. Mol. Cell. Biol. 25: 3553-
3562.

Taylor, J.H. 1977. Increase in DNA replication sites in cells held at the beginning of S phase. Chromosoma 62: 291-300.

Tsao, C.C., Geisen, C., and Abraham, R.T. 2004. Interaction between human MCM7 and Rad17 proteins is required for replication checkpoint signaling. EMBO J. 23: 4660-4669.

Woodward, A.M., Gohler, T., Luciani, M.G., Oehlmann, M., Ge, X., Gartner, A., Jackson, D.A., and Blow, J.J. 2006. Excess Mcm2-7 license dormant origins of replication that can be used under conditions of replicative stress. J. Cell Biol. 173: 673-683.

Yankulov, K., Todorov, I., Romanowski, P., Licatalosi, D., Cilli, K., McCracken, S., Laskey, R., and Bentley, D.L. 1999. MCM proteins are associated with RNA polymerase II holoenzyme. Mol. Cell. Biol. 19: 6154-6163. 


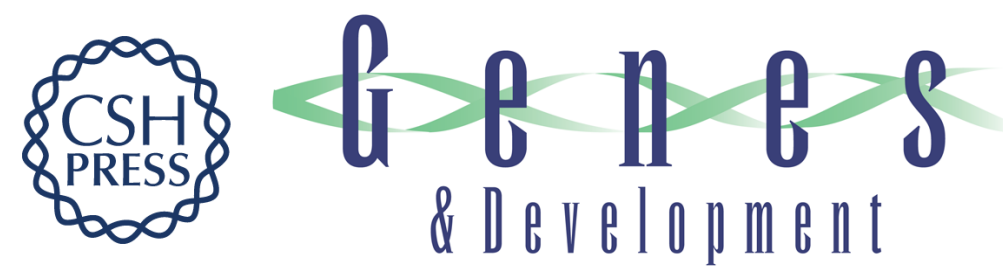

\section{Dormant origins licensed by excess Mcm2-7 are required for human cells to survive replicative stress}

Xin Quan Ge, Dean A. Jackson and J. Julian Blow

Genes Dev. 2007, 21:

Access the most recent version at doi:10.1101/gad.457807

Supplemental http://genesdev.cshlp.org/content/suppl/2007/11/28/21.24.3331.DC1
Material

References This article cites 55 articles, 29 of which can be accessed free at:

http://genesdev.cshlp.org/content/21/24/3331.full.html\#ref-list-1

License

Email Alerting

Receive free email alerts when new articles cite this article - sign up in the box at the top

Service

right corner of the article or click here.

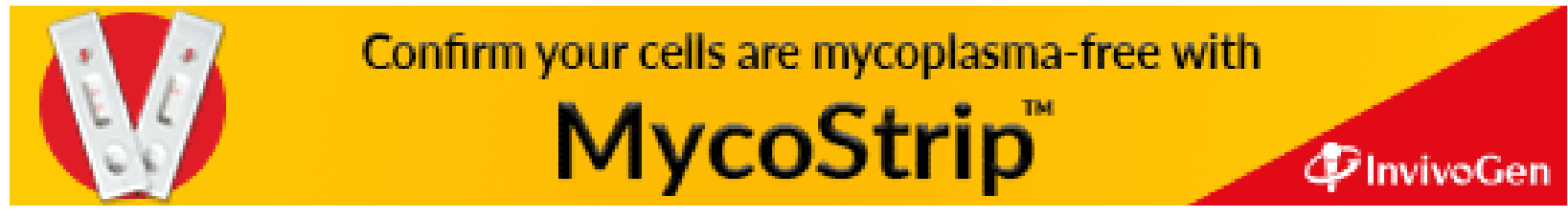

